\title{
Photoconjugation of molecularly imprinted polymer nanoparticles for surface enhanced Raman detection of propranolol
}

Tripta Kamra ${ }^{a, c}$, Changgang $X u^{a}$, Lars Montelius ${ }^{b, s}$, Joachim Schnadt ${ }^{c}$, Samurdhi A. Wijesundera $^{d}$, Mingdi Yan ${ }^{d, e}$, Lei Ye ${ }^{a *}$

${ }^{a}$ Division of Pure and Applied Biochemistry, Lund University, Box 124, 22100 Lund, Sweden

${ }^{\mathrm{b}}$ Division of Solid State Physics, Lund University, Box 118, 22100 Lund, Sweden

${ }^{c}$ Division of Synchrotron Radiation Research, Lund University, Box 118, 22100 Lund, Sweden

d Department of Chemistry, University of Massachusetts Lowell, 1 University Ave., Lowell, MA 01854, USA

${ }^{\mathrm{e}}$ Department of Chemistry, KTH - Royal Institute of Technology, Teknikringen 30, 10044 Stockholm, Sweden

$\S$ Present address: International Iberian Nanotechnology Laboratory, Avenida Mestre José Veiga s/n, 4715-330 Braga, Portugal

\section{Corresponding Author:}

* Lei Ye, Email: lei.ye@,tbiokem.lth.se Tel.: +46 462229560. 


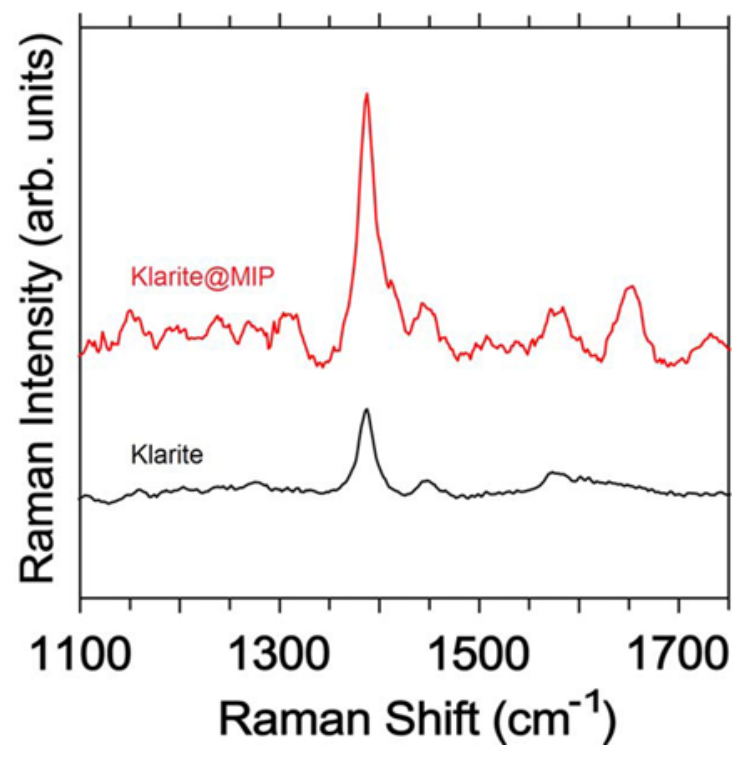

Figure S1. Raman spectra of $3.85 \times 10^{-5} \mathrm{M}$ propranolol obtained on MIP-coated Klarite (top) and on Klarite (bottom). The spectra were background subtracted and smoothened using the Savitzky-Golay method. 